ISSN: $1130-3743$

DOI: http://dx.doi.org/10.14201/teoredu2014261161186

\title{
LA TUTORÍA UNIVERSITARIA EN EL ESCENARIO DEL ESPACIO EUROPEO DE EDUCACIÓN SUPERIOR: PERFILES ACTUALES
}

\section{University tutorials in the setting of the European Higher Education Area: current profiles}

\section{Tutorat universitaire dans l'Espace Européen de l'Enseignement Supérieur: profils actuels}

\author{
Carolina FERnÁnDEZ-SALINERO Miguel \\ Universidad Complutense de Madrid. Facultad de Educación. Centro de \\ Formación del Profesorado. Departamento de Teoría e Historia de la Educación. \\ C/ Rector Royo Villanova, s/n. 28040 Madrid. Correo-e: cfernand@edu.ucm.es
}

Fecha de recepción: enero de 2014

Fecha de aceptación: abril de 2014

Biblid [(1130-3743) 26, 1-2014, 161-186]

RESUMEN

En el nuevo escenario promovido por el Espacio Europeo de Educación Superior, la orientación y la tutoría universitarias adquieren más importancia que nunca. Entendemos la tutoría como una parte de la responsabilidad docente, en la que se establece una interacción más personalizada entre el profesorado y el alumnado, entre docente experimentado y docente novel o entre estudiante y estudiante, cuyo objetivo es guiar el aprendizaje, adaptándolo a las condiciones individuales y al estilo de aprender de los sujetos involucrados. Ahora es el momento de plantear sistemas de guía y tutela para los estudiantes -durante el proceso de formación y en sus primeros pasos profesionales- y para el profesorado novel. Entre dichos siste- 
LA TUTORÍA UNIVERSITARIA EN EL ESCENARIO DEL ESPACIO EUROPEO DE EDUCACIÓN SUPERIOR: PERFILES ACTUALES

mas se encuentran el coaching ejercido por el docente o el mentoring, este último desarrollado a través de la tutoría entre iguales, la tutoría profesional en los centros de prácticas o la tutoría ejercida por profesorado experimentado hacia profesorado novel.

Palabras clave: coaching, Espacio Europeo de Educación Superior, mentoría entre iguales, mentoring, tutoría, tutoría experimentada, tutoría entre iguales, tutoría profesional.

\section{SUMMARY}

In the new setting promoted by the European Higher Education Area, university guidance and tutorials have become more important than ever. We understand tutorials as part of the teaching responsibility in which a more personal interaction between professor and student, professor and novice teacher, or student and student is established, and whose goal is to guide learning according to the individual characteristics and learning styles of the individuals involved. Now is the time to set up guidance and tutorials systems for students -both during the training process and in their first professional steps- and for novice teachers also. Among such systems we can mention professor coaching, peer mentoring, professional tutoring in training centres or mentoring of an experienced university professor on the novice teacher.

Key words: coaching, European Higher Education Area, mentoring, tutoring, peer mentoring, experienced tutoring, peer tutoring, professional tutoring.

\section{SOMMAIRE}

Dans l'Espace Européen de l'Enseignement Supérieur, l'orientation est très important. L'orientation est la responsabilité de l'enseignant et facilite la relation entre l'enseignant et l'élève, entre le professeur et les enseignants débutants ou entre élève et élève. L'objectif est de guider l'apprentissage, en fonction des caractéristiques individuelles et la façon d'apprendre de chaque personne. Maintenant, il devrait y avoir de nouveaux systèmes d'orientation pour les étudiants -à la fois académiques et professionnelles- et pour les enseignants. Ces systèmes de guidage peuvent être: coaching exercé par l'enseignant, le mentorat exercé par des étudiants, le mentorat exercé par les enseignants des centres de pratique ou le mentorat par des professeurs experimentés exercées sur les enseignants débutants.

Mots clés: coaching, Espace Européen de l'Enseignement Supérieur, mentorat expérimenté, mentoring, mentorat par les pairs, mentorat professionnel, tutorat, tutorat par les pairs. 


\section{Planteamientos intRODUCTORIOS}

La educación, hoy en día, debe afrontar la sensación de vértigo que se vive ante el dilema de la globalización. Se atribuye a la educación la misión de permitir a todos los ciudadanos el hacer fructificar todos sus talentos y todas sus capacidades de creación, lo que implica que cada uno pueda responsabilizarse de sí mismo y realizar su proyecto personal (Delors, 1997). Nos enfrentamos ahora a un proceso de transición en el que se hace necesario revalorizar los aspectos éticos y culturales de la educación, y para ello dar a cada uno los medios para comprender al otro en su particularidad y comprender el mundo en su curso caótico hacia una cierta unidad. En este escenario, la educación superior debe brindar competencias básicas a sus estudiantes para incorporarles a una sociedad y a un mundo productivo que está en constante cambio a nivel tecnológico, científico, político, económico y social. Los estudiantes de hoy demandan servicios educativos que presenten diferencias respecto a sus necesidades, trayectorias de formación y aspiraciones. Al ingresar en los diferentes Grados universitarios, los estudiantes están consolidando su transición adolescencia-juventud, a la que se suma la transición de nivel académico, donde cambian espacios, normas y cultura escolar. El tutelaje personalizado en estos momentos disminuye la ansiedad, favorece la integración a la nueva institución y mejora las condiciones para el aprendizaje.

Es precisamente en este nuevo escenario, promovido por el Espacio Europeo de Educación Superior (EEES), en el que la orientación y la tutoría universitarias adquieren más importancia que nunca. Ahora es el momento de plantear sistemas de guía y tutela para los estudiantes -durante el proceso de formación y en sus primeros pasos profesionales-, pero también para el profesorado, que se basen en el desarrollo autónomo, tanto de las competencias técnicas como de las personales.

\section{DEFINICIÓN DE TUTORÍA UNIVERSITARIA}

Entendemos la tutoría como una parte de la responsabilidad docente, en la que se establece una interacción más personalizada entre el profesorado y el alumnado (aunque también, entre docente y docente o entre estudiante y estudiante), con el objetivo de guiar el aprendizaje de dicho alumnado, adaptándolo a sus condiciones individuales y a su estilo de aprender, de modo que cada estudiante alcance el mayor nivel de dominio posible. La tutoría es, por tanto, un componente inherente de la formación universitaria. Comparte sus fines y contribuye a su logro, facilitando la adaptación a la universidad, el aprendizaje y el rendimiento académico, la orientación curricular y la orientación profesional. Y, cada vez más, debe concebirse como una actividad sistemática, intencional, correctamente articulada y programada. Para ello, se requiere dotarla de una estructura de funcionamiento y concederle un lugar destacado en la planificación de las actividades académicas propias del quehacer de la universidad. Sólo así puede llegar a ser un elemento importante de calidad (Cano, 2008). 
LA TUTORÍA UNIVERSITARIA EN EL ESCENARIO DEL ESPACIO EUROPEO DE EDUCACIÓN SUPERIOR: PERFILES ACTUALES

La tutoría, así entendida, se considera como una acción nuclear dentro del conjunto de acciones impulsadas en todas las universidades para ayudar a resolver las encrucijadas entre la cantidad y la calidad, entre la masificación y la personalización, entre la gestión del profesorado y la gestión del alumnado, entre el énfasis por el resultado y el énfasis en el proceso (Rodríguez Espinar, 2004). Y su puesta en práctica demanda un nuevo paradigma universitario, que sustituya el modelo de formación-instrucción basado en la transmisión de contenidos por otro modelo de educación-profesionalización, con un aumento sustancial de la comunicación e interacción entre docentes y estudiantes, entre los propios docentes y entre los propios estudiantes, entre empresas y estudiantes y, en definitiva, entre la universidad y la sociedad. Que implique adoptar, en consecuencia, un método centrado en el estudiante frente a otros centrados en el profesorado o la materia.

En estos términos, la tutoría universitaria debe concebirse como un proceso que permita planificar de manera preventiva determinadas actuaciones educativas, o intervenir si fuera preciso para lograr los objetivos de desarrollo competencial individual y profesional deseados (Martínez González, 2011). La oferta de una tutoría universitaria innovadora puede ser una herramienta eficaz para la construcción guiada del aprendizaje de los estudiantes (e incluso de los docentes noveles) y de su desarrollo autónomo, mediante la adquisición, integración y puesta en práctica de un conjunto amplio de competencias (generales y específicas) para la acción, que han de poseer y saber aplicar todos los universitarios como certificación de su capacidad, formación y valía personal (Cano, 2009).

Las características de la tutoría universitaria, desde esta perspectiva, serían las siguientes (Rodríguez Espinar, 2004, 28):

- Es una acción de orientación dirigida a impulsar y facilitar el desarrollo integral de los estudiantes en su dimensión intelectual, afectiva, personal, social y profesional, en línea con un planteamiento de calidad desde la perspectiva del que aprende; así como del profesorado principiante que comienza su labor como docente universitario.

- La tutoría contribuye a personalizar la educación universitaria.

- La atención a la persona tutorada es un elemento clave de calidad.

- La tutoría canaliza y dinamiza las relaciones de los individuos tutorados con los diferentes segmentos de atención a los mismos, tanto de carácter administrativo, docente, organizativo y de servicios.

- La tutoría permite la integración activa de las personas tutoradas en la institución.

- Favorece la formación de un verdadero espíritu, estilo y perfil universitario, y encamina al individuo tutorado hacia la madurez personal y el crecimiento intelectual y científico (García Nieto, 2008). 
LA TUTORÍA UNIVERSITARIA EN EL ESCENARIO DEL ESPACIO EUROPEO DE EDUCACIÓN SUPERIOR: PERFILES ACTUALES

Dentro de un modelo de formación holístico, las dimensiones de intervención de la tutoría incluyen las áreas relacionadas con el desarrollo personal del universitario, en el sentido siguiente:

- Dimensión intelectual-cognitiva: incluye la estrategia de "aprender a aprender", es decir, enfrentar al universitario a problemas para que diseñe soluciones.

- Dimensión afectivo-emocional: supone potenciar el dominio de habilidades sociales, el autoconocimiento, el reforzamiento de la autoimagen.

- Dimensión social: comprende las habilidades para integrarse en un grupo social y la participación en el mismo de manera activa.

- Dimensión profesional: supone que el universitario tenga claro el itinerario de su proyecto de vida.

Cada una de estas dimensiones se desarrolla en diferentes formas (académica, personal y profesional), a distinto nivel (inicial, de apoyo o de seguimiento), con distinto carácter (obligatoria o voluntaria, virtual o presencial) y diversas manifestaciones (individual, en grupo, entre iguales); todas ellas complementarias, como elemento fundamental y anexo al ejercicio de la función docente del profesorado o de la función de apoyo de sus propios compañeros de estudios, cuya finalidad o propósito se concreta en ayudar a los estudiantes a conseguir los objetivos y competencias propios de la profesión para la que se están preparando (Cano, 2008). En este sentido, podemos identificar diferentes tipos de tutorías:

TABla 1. Rodríguez EsPinAR, 2004, 31-32

\begin{tabular}{|c|c|}
\hline CRITERIOS & TIPOS DE TUTORÍA \\
\hline $\begin{array}{l}\text { En relación con } \\
\text { los servicios de } \\
\text { orientación }\end{array}$ & $\begin{array}{l}\text { Modelo de tutoría puro: el programa de tutorías funciona de modo independiente } \\
\text { de los servicios de orientación de la institución. } \\
\text { Modelo mixto: el modelo de tutoría se organiza con apoyo de los servicios de } \\
\text { orientación. }\end{array}$ \\
\hline $\begin{array}{l}\text { En cuanto al } \\
\text { contenido de } \\
\text { las tutorías }\end{array}$ & $\begin{array}{l}\text { Tutoría de materia: se ocupa de orientar al estudiante sobre temas relativos a los } \\
\text { contenidos disciplinares que el profesorado expone o explica en clase, o sobre } \\
\text { temas de trabajos relacionados con la materia. } \\
\text { Tutoría de prácticas: tiene como finalidad proporcionar al estudiante el espacio para } \\
\text { desarrollar habilidades prácticas y herramientas para su desempeño profesional. } \\
\text { Tutoría de proyecto: se dirige a asesorar y apoyar todo el trabajo del proyecto del } \\
\text { estudiante. } \\
\text { Tutoría de asesoramiento personal: se trata de una tutoría especializada para el } \\
\text { tratamiento de la situación particular del estudiante. Esta tutoría debe estar bajo la } \\
\text { responsabilidad de expertos en la intervención psicopedagógica, o bien en manos } \\
\text { de los servicios de orientación de la universidad. }\end{array}$ \\
\hline $\begin{array}{l}\text { En cuanto a la } \\
\text { figura del tutor }\end{array}$ & $\begin{array}{l}\text { Profesor/a tutor/a: esta tarea es asumida por el docente, tanto respecto a los } \\
\text { estudiantes, como de un docente experimentado a otro novel. } \\
\text { Tutoría de iguales (peer tutoring): los estudiantes de cursos superiores son los } \\
\text { encargados de orientar y asesorar a sus compañeros, de manera exclusiva o de } \\
\text { forma complementaria. }\end{array}$ \\
\hline
\end{tabular}


LA TUTORÍA UNIVERSITARIA EN EL ESCENARIO DEL ESPACIO EUROPEO DE EDUCACIÓN SUPERIOR: PERFILES ACTUALES

\begin{tabular}{|l|l|}
\hline \multicolumn{1}{|c|}{ CRITERIOS } & \multicolumn{1}{c|}{ TIPOS DE TUTORÍA } \\
\hline $\begin{array}{l}\text { Con relación } \\
\text { al tiempo }\end{array}$ & $\begin{array}{l}\text { Tutoría de curso: se refiere al seguimiento del estudiante en un tramo de su trayecto } \\
\text { formativo. } \\
\text { Tutoría de carrera o de itinerario académico: se refiere a un seguimiento del } \\
\text { estudiante a lo largo de los estudios universitarios, en cuestiones generales relativas } \\
\text { a itinerarios curriculares, a la adaptación a la vida universitaria, a la mejora del } \\
\text { rendimiento o a las salidas profesionales. }\end{array}$ \\
\hline $\begin{array}{l}\text { En cuanto a los } \\
\text { destinatarios }\end{array}$ & $\begin{array}{l}\text { Tutoría individual: acción personalizada, útil para tratar aspectos personales de } \\
\text { carácter individual. } \\
\text { Tutoría grupal: cuando en grupo se tratan temas comunes a los integrantes del } \\
\text { mismo. }\end{array}$ \\
\hline
\end{tabular}

En la actualidad coexisten tres grandes modelos de tutoría (Rodríguez Espinar, 2004):

- Modelo académico (teaching): más vinculado a la tradición continental, en el que la acción docente se restringe a informar u orientar sobre asignaturas, en un ambiente de máxima autonomía y libertad de todos los miembros de la comunidad universitaria.

- Modelo de desarrollo personal (counseling): más relacionado con el modelo anglosajón, en el que el objetivo es el desarrollo integral del estudiante y, por tanto, la tutoría abarca ámbitos más allá del estrictamente académico, para adentrarse en cuestiones profesionales y personales.

- Modelo de desarrollo profesional (career advising): en el que la figura del tutor de la universidad se ve completada por la del tutor en el lugar de trabajo, donde se desarrollan las prácticas en situaciones reales y en las que el principal objetivo es el desarrollo de competencias personales, académicas y profesionales para que el perfil del estudiante se ajuste al máximo a los requerimientos del puesto profesional que se va a ver obligado a desempeñar.

Con este planteamiento de la tutoría en la universidad descubrimos que es el estudiante el verdadero protagonista del proceso de orientación, ya sea como objetivo directo de intervención (a través del asesoramiento del docente universitario y del profesional del entorno de las prácticas, así como del apoyo de los compañeros de cursos superiores), o como objetivo indirecto (derivado de los aprendizajes que un docente novel adquiere gracias al apoyo de otro docente experimentado). En cada caso, el perfil del tutor será diferente y estará asumido por distintas personas. Este es el punto que nos parece fundamental y que vamos a tratar a continuación. 


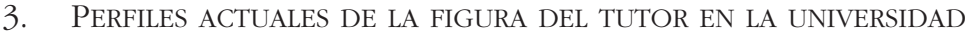

En este contexto, podemos definir al tutor como el "docente -y también estudiante- que tutela la formación humana y científica de un estudiante y le acompaña en sus procesos de aprendizaje, (Michavila y Delgado, 2003), y cuya actividad está encaminada a propiciar un proceso madurativo permanente, a través del cual el estudiante universitario logre obtener y procesar información correcta sobre sí mismo y su entorno, dentro de planteamientos intencionales de toma de decisiones razonadas; afiance su autoconcepto por medio de experiencias vitales en general y laborales en particular; y despliegue las habilidades y actitudes precisas para conseguir integrar el trabajo dentro de un proyecto de vida global (Cano, 2008, 19).

En palabras de Novo (2007, 356-357):

El énfasis educativo, en este enfoque, no se pone sólo en atender a las capacidades y destrezas individuales, o preparar profesionales para el mercado, sino en introducir en el corazón del acto educativo los problemas de la sociedad, desde la escala local hasta la global, fomentando la responsabilidad colectiva, y potenciando así el carácter transformador y liberador que puede tener la educación.

En este sentido, la construcción de las identidades se despliega en un complejo entramado entre los individuos y las comunidades, entre los sujetos y las prácticas socioculturales y profesionales en que se desenvuelven, generándose procesos de autoconciencia y de autodeterminación. Las instituciones educativas universitarias, como instituciones específicas de educación, han de establecer sin ambages su compromiso con la profunda tarea de educar, con su inestimable labor de ayuda a la formación en y para la autonomía y la responsabilidad (Argos et al., 2011).

Y los tutores deben ser los que faciliten al estudiante una ayuda, basada en una relación personalizada, que se dirige a conseguir sus objetivos académicos, profesionales y personales a través del uso de la totalidad de recursos institucionales y comunitarios. En este sentido, el profesorado universitario, ante el EEEs, ha de añadir a sus funciones tradicionales como docente e investigador una tercera función como tutor. Como señalan Michavila y Delgado (2003), encontramos tres grandes funciones docentes: una función instructiva, relacionada con la transmisión del saber; una función investigadora, mediante la que debe contribuir al avance de la ciencia y a la búsqueda de nuevos saberes; y una función formativa o de tutoría, a través de la cual debe desarrollar en los estudiantes sus actitudes, hábitos y destrezas. Funciones que los estudiantes de cursos superiores pueden ejercer, también, dentro de un sistema de tutoría bien planteado, con respecto a sus compañeros de cursos inferiores.

Debemos identificar, a este respecto, las cualidades específicas que todo tutor debe tener. Son varias y de diferente tipo. A continuación vamos a realizar una breve clasificación de las mismas. 


\section{TABLA 2}

Cualidades humanas: actitudes que posibilitan la relación profunda, rica y eficaz con los otros. No son innatas, pero pueden mejorarse con el ejercicio.

Cualidades científico-técnicas

definen el saber y el saberhacer del tutor. Son el conjunto de destrezas y técnicas que se adquieren por medio de la formación continua (formal, no formal o informal), aunque en cierta medida dependen de las cualidades humanas.

Elaboración propia.

Una vez definido el tutor en la universidad y delimitadas sus características fundamentales, conviene precisar a continuación los perfiles actuales del tutor, que le otorgan a la tutoría ese carácter integrador y a su responsable esa labor de acompañamiento que todo estudiante necesita.

\subsection{Coaching: la figura del docente como coach}

El docente que ejerce como tutor en la universidad debe jugar el papel de un coach (Hashuel, 2010). El coaching se concibe como una experiencia formativa sustentada en la confianza mutua entre tutor y estudiante y busca la dinamización 
LA TUTORÍA UNIVERSITARIA EN EL ESCENARIO DEL ESPACIO EUROPEO DE EDUCACIÓN SUPERIOR: PERFILES ACTUALES

y el asesoramiento de un docente hacia un estudiante, dentro del entorno académico. La mayéutica es su filosofía.

La educación que aspira a formar sujetos autónomos lo primero que transmite a los educandos es que la expectativa de alcanzar la autonomía estriba en la adopción de un particular proyecto de situación y relación comprometida con el mundo (Ruiz Corbella et al., 2011, 16). Con esta finalidad, el tutor de la universidad, en su papel de coach, debe seguir las siguientes etapas (De Mariano, 1999, 31-32):

- Establecer la relación: la acción comienza cuando hacemos nuestros los problemas del otro y buscamos la confidencialidad a través de la apertura, transparencia y comunicación.

- Escuchar propuestas: se trata de favorecer cualquier tipo de comunicación y aprovecharla para entender más al otro.

- Aceptar. el objetivo es acortar distancias entre los problemas del otro y nuestros propios problemas; relativizando las diferencias y aproximando las posiciones.

- Apreciar debemos ir convirtiéndonos en un confidente que, paulatinamente, va conociendo cada vez mejor a su compañero.

- Aprender tenemos ahora que hacer preguntas sobre la concepción del mundo del otro y percibir con él.

- Compartir información: llega un momento en que se produce el feedback, que es simplemente comunicación de información no juzgada.

- Decidir. el aprender y adaptarse a los cambios y abrirse a las personas constituye lo que denominamos retos, y la actitud de abrirse a esos retos es lo que facilita el éxito de los estudiantes.

- Actuar. en este momento estamos transformando el mundo y abriéndonos a progresar en él, viviendo entre todos un brainstorming para que las ideas de todos nos ayuden.

- Visionar el sistema: a partir de aquí entramos en el mundo de las nuevas concepciones y de las prioridades que formulan nuestras concepciones originales y que una vez que hemos aprendido hacen que veamos el mundo de otra forma.

- Buscar la convergencia: en este momento se ve la convergencia de los criterios de las personas que no son tan diferentes cuando se enriquecen.

- Dar prioridad: se produce cuando el tutor puede dar un consejo, y nuestra visión se enfoca y nos ilustra en las prioridades, que además nos indican qué camino tomar. Cuando las prioridades las tenemos claras, es cuando las oportunidades aparecen, pues ya somos capaces de verlas. Y es cuando la autonomía entra en juego.

Durante este proceso, el estudiante va configurando y diseñando su Proyecto Profesional y Personal, que podríamos denominar mejor como "anteproyecto", por cuanto debe ser abierto y estar sujeto a múltiples y continuadas redefiniciones a partir de sus experiencias formativas (Hernández Franco y Torres, 2005, 26). 
LA TUTORÍA UNIVERSITARIA EN EL ESCENARIO DEL ESPACIO EUROPEO DE EDUCACIÓN SUPERIOR: PERFILES ACTUALES

Un tutor que actúe como coach debe cumplir las siguientes funciones básicas dentro de un sistema integrado de tutoría (Hernández Franco y Torres, 2005, 34-35):

- Función de información: el coach debe estar siempre informado y actualizado para poder responder a los requerimientos del estudiante.

- Función de mediación: entre los estudiantes y otros servicios, recursos o instancias de la universidad.

- Función de seguimiento y orientación académica: el coach debe establecer espacios para que el estudiante reflexione sobre su trayectoria académico-profesional y donde pueda analizar las decisiones que deba ir tomando a lo largo de sus estudios.

- Función formativa: el tutor que actúa como coach debe ayudar al estudiante a descubrir posibles vacíos formativos y a buscar con él soluciones dentro o fuera de la universidad.

- Función de atención a las necesidades de carácter personal: se trata de convertirse en el "rostro" de la institución educativa con el que el estudiante puede contar, y de atender a la persona que "hay detrás" del estudiante.

- Función de coordinación horizontal: entre el profesorado-tutor de un curso que imparte una misma asignatura en diferentes grupos; entre el profesorado-tutor de diferentes asignaturas que se imparten en un mismo curso; entre el profesorado-tutor que imparte asignaturas en cursos sucesivos; así como, entre el profesorado-tutor y los agentes de orientación de la universidad.

- Función de ayuda en la transición al empleo: la tutoría debe ofrecer al estudiante espacios donde pueda pensar y reflexionar como futuro profesional. El tutor debe ayudar al estudiante a preparar su proyecto profesional, de manera que sea él mismo el que conduzca la transición de la universidad al empleo; analizando su potencial profesional y estableciendo medidas para incrementarlo y dirigirlo hacia donde su preparación y vocación le puedan ser más útiles.

\subsection{Mentoring: la tutoría entre iguales, el tutor de prácticas y los docentes experimentados como mentores}

El tutor se convierte en un mentor ${ }^{1}$, en una persona con más conocimientos, habilidades y experiencia que se pone en contacto con otra que necesita de los mismos para su crecimiento (Murray, 2001). Se encarga de guiar y orientar el

1. La figura del mentor nace en la cultura griega (Mentor fue el instructor elegido por Ulises por su experiencia, y no por su saber académico, para Telemaco, su hijo), pasando por la figura del maestro y el aprendiz en los oficios de la Edad Media, hasta el tutor como figura en la pedagogía actual. 
LA TUTORÍA UNIVERSITARIA EN EL ESCENARIO DEL ESPACIO EUROPEO DE EDUCACIÓN SUPERIOR: PERFILES ACTUALES

desarrollo de las capacidades, competencias y actitudes del otro, más acordes con su potencial. Debe poseer habilidades de comunicación (empatía y asertividad) y favorecer el aprendizaje, generando una relación de confianza. La mentoría, en este sentido, puede ser entendida como un proceso de ayuda al aprendizaje con distintas variantes y modelos de desarrollo (natural, intencionada, de tránsito, etc.) (Valverde et al., 2003-2004).

Un elemento definitorio del mentoring es la confidencialidad de todo el proceso, que requiere, de mentor y mentorizado, el compromiso con el cumplimiento de los acuerdos establecidos y la rigurosidad en el planteamiento de la actividad propuesta. La claridad, implicación y seguimiento deben primar aquí, asegurando de este modo la correcta y eficaz consecución de las metas establecidas (Fernández-Salinero, 2008, 144). El compromiso, además de con uno mismo, le vincula con los demás, puesto que con ellos convive y a ellos afectan las consecuencias de sus actos u omisiones (Ruiz Corbella et al., 2011, 25).

Un proceso de mentoring de estas características se lleva a cabo por el mentor del siguiente modo (Díaz, 2000, 73):

1. Identificación del grupo o de la persona a tutorar.

2. Valoración de cada individuo en relación con los requisitos necesarios en el proceso de enseñanza-aprendizaje (mentoring natural).

3. Orientación a los involucrados, aportándoles pautas de actuación sobre qué pueden esperar y sus responsabilidades (mentoring formal).

4. Análisis de la percepción de los mentorizados, de sus fortalezas y necesidades de desarrollo, así como de sus aspiraciones académicas y profesionales (mentoring situacional).

5. Establecimiento de reuniones de revisión de los progresos alcanzados, discusión sobre proyectos o actividades actuales y acuerdo sobre nuevos objetivos (mentoring de supervisión).

6. Evaluación del impacto en el mentorizado, promoviendo un feedback para futuras relaciones y analizando el nivel de autonomía profesional alcanzado por el mismo.

Se observa, en este sentido, la importancia que tiene para el mentor utilizar diferentes sistemas de comunicación interpersonal (facilitador, colaborador, directivo), en relación con distintos tipos de mentorizados (Rowley, 1999). Y se descubre la existencia de diferentes perfiles de mentor, ya nos refiramos a la tutoría entre iguales, a la tutoría profesional del estudiante en los centros de prácticas o a la tutoría del docente universitario experimentado sobre el profesorado novel. Cada una de ellas vamos a definirla más detalladamente a continuación.

- La tutoría entre iguales

Los programas de mentoría entre iguales (peer mentoring) pretenden favorecer la adaptación a la institución universitaria del alumnado de nuevo acceso, facilitándole acogida, integración, información, asesoramiento y orientación para 
LA TUTORÍA UNIVERSITARIA EN EL ESCENARIO DEL ESPACIO EUROPEO DE EDUCACIÓN SUPERIOR: PERFILES ACTUALES

su integración académica y social, así como para conseguir el éxito en sus estudios universitarios. Se presenta como una buena estrategia para desarrollar conjuntamente competencias generales y específicas dentro de un contexto de aprendizaje colaborativo y de un clima de confianza (Velasco y Benito, 2011).

De manera más específica, los objetivos que se pretenden alcanzar con este tipo de mentoría serían los siguientes (Sánchez Ávila et al., 2007):

- Dentro de la orientación académica, proporcionar al mentorizado la ayuda necesaria para abordar con éxito las diferentes materias del curso desde la experiencia del mentor, buscando la mejora en la adquisición de conocimientos sobre el entorno académico, el incremento del rendimiento y la satisfacción en los estudios.

- Dentro de la orientación social, ayudar al estudiante de nuevo ingreso en su adaptación a la universidad, atendiendo a la diversidad, informándole sobre los recursos de tipo social a los que puede acudir y promoviendo un mayor autoconcepto, autoestima y autoeficacia del mentorizado.

- Dentro de la orientación administrativa, informar al estudiante de nuevo ingreso acerca de los procedimientos administrativos generales.

Las fases de actuación de un programa de mentoría de estas características, una vez revisadas diferentes experiencias implementadas en universidades españolas e internacionales ${ }^{2}$, podríamos sintetizarlas en las siguientes (Velasco y Benito, 2011):

1) Plan de difusión y presentación del programa: inicialmente de manera virtual en la página web de las universidades o de sus servicios de orientación. Posteriormente, en sesiones presenciales de gran grupo al comienzo de curso, como las sesiones de bienvenida a los estudiantes de nuevo ingreso, y después en pequeños grupos, primero a los mentores y luego, a través de éstos, a los mentorizados.

2) Captación de estudiantes participantes: tanto de mentores como de mentorizados. Los primeros, entre aquellos con mejor expediente académico y con buenas habilidades interpersonales. Los segundos, entre aquellos estudiantes de nuevo ingreso o procedentes de programas

2. Entre las experiencias consultadas podemos destacar las siguientes: el Proyecto Souestututor de la Universidad Complutense de Madrid, el Programa Brújula de la Universidad Europea de Madrid, el Proyecto Mentor de la Universidad Politécnica de Madrid, el Proyecto Compañero de la Universidad de Cádiz, el Programa Mentor de la Universidad de Burgos o el Programa de Mentoring o Tutorías entre iguales de la Universidad Rey Juan Carlos. Muchas de ellas inscritas, desde 2008, en una Red de Mentoría Universitaria, coordinada por la Universidad Politécnica de Madrid, que trata de agrupar las iniciativas de mentoría llevadas a cabo en diferentes universidades españolas. Y a nivel internacional el Peer Education Program de la Universidad de Nueva York, el Peer Tutoring Programa de la Universidad de Harvard o la experiencia de Tutorías entre Pares (TEPs) de la Universidad de la República de Uruguay. 
LA TUTORÍA UNIVERSITARIA EN EL ESCENARIO DEL ESPACIO EUROPEO DE EDUCACIÓN SUPERIOR: PERFILES ACTUALES

de intercambio y movilidad con interés en participar voluntariamente en esta experiencia.

3) Formación de mentores: los estudiantes seleccionados como mentores reciben un curso de capacitación orientado a desarrollar competencias transversales, que incluyen habilidades sociales, trabajo en equipo, empatía y escucha activa, motivación, innovación y creatividad, planificación, responsabilidad con la tarea, autoconfianza, toma de decisiones, iniciativa, orientación a resultados y solución de problemas. Y que incluye también información relativa a los diversos servicios de la universidad (facultades, bibliotecas, actividades deportivas, becas, cursos, etc.).

4) Constitución de grupos: habitualmente, cada mentor tiene a su cargo entre tres y seis mentorizados, pero esto depende del número de mentores y mentorizados que se apunten de manera voluntaria al programa, por lo que puede desarrollarse mentoring individual en algunos casos.

5) Seguimiento: se suelen llevar a cabo reuniones periódicas entre el docente coordinador y los mentores (una al mes aproximadamente) y entre el mentor y sus mentorizados (una a la semana o cada quince días, aproximadamente), siendo la duración global de este tipo de programas de unos seis meses.

6) Elaboración de informes y evaluación: por parte del mentor, en el que se recoge, a modo de diario reflexivo, su percepción del desarrollo de la labor realizada; y por parte del docente coordinador, quien a través de cuestionarios de valoración respondidos por mentores y mentorizados, obtiene información sobre el grado de satisfacción alcanzado por todos los participantes, los problemas surgidos, el material aportado, la coordinación del grupo, etc.

7) Reflexión y propuestas de mejora: una vez finalizada la experiencia y analizados los resultados, se toman decisiones de continuidad y mejora de dicha experiencia para su desarrollo en cursos siguientes.

Su estructura se apoya en la siguiente cadena jerárquica:

1. Los docentes tutores son los coordinadores del programa y están encargados de supervisar el correcto funcionamiento del programa y de solucionar los problemas que se planteen.

2. Los mentores son los estudiantes de cursos superiores que aceptan participar en el programa y que reciben inicialmente formación como mentores, potenciando en ellos habilidades sociales, de relación, orientación y liderazgo.

3. Los mentorizados son estudiantes de nuevo ingreso que aceptan participar en el programa. 
LA TUTORÍA UNIVERSITARIA EN EL ESCENARIO DEL ESPACIO EUROPEO DE EDUCACIÓN SUPERIOR: PERFILES ACTUALES

Podemos hacer mención de diferentes tipos de mentoría:

\subsubsection{Mentoría presencial}

- Mentoring de grupo formal, planificado, intencional y sistemático, en el que el mentor, estudiante de cursos superiores, mentoriza a varios compañeros de primer curso o procedentes de programas de intercambio internacional. Se trata de un mentoring entre iguales, ya que ambos grupos tienen un mismo estatus académico (el de alumnado), si bien la experiencia y los conocimientos de los mentores resultan superiores a los de los mentorizados.

- Mentoring de grupo informal, que es aquel que se realiza de manera espontánea entre compañeros de clase, normalmente entre aquellos que poseen un mayor dominio de una materia y aquellos otros que adolecen de dicho conocimiento.

- Mentoring individual, que es aquel que puede ser más formalizado y tendría similares características que el mentoring de grupo formal. Y aquel otro que tiene un carácter más espontáneo y que se asemejaría al de grupo informal.

\subsubsection{Mentoría virtual}

- $\quad$ Mentoring electrónico entre pares, en el cual se utilizan entornos virtuales de comunicación que maximizan el aprendizaje en red y aprovechan la ventaja de la flexibilidad espacial y temporal que ofrece el e-mentoring, acomodándose a las preferencias individuales de los mentorizados. En este tipo de mentoría, los mentores deben ser capaces de crear una sensación de "presencia social", con el fin de crear apego relacional y "química electrónica" (Risquez, 2011). Y los mentorizados pueden agruparse o participar en el proceso de mentoring de manera individual.

\subsubsection{Mentoría combinada}

- Mentoring electrónico que se complementa con la realización de sesiones presenciales, cuyo objetivo es favorecer un contacto más personal, tanto grupal como individual, a la hora de resolver dudas o problemas particulares.

Con todo ello, lo que se pretende es convertir la mentoría en una estrategia de innovación educativa eficaz y eficiente dentro de la universidad, para conseguir una buena y rápida adaptación del alumnado al entorno universitario. Esta orientación consigue, además, que tanto mentor como mentorizado desarrollen conjuntamente competencias generales, como la responsabilidad, el trabajo en equipo, la planificación o las habilidades comunicativas, aunque cada uno de ellos en distintos niveles (Velasco y Benito, 2011). 


\section{- La tutoría profesional del estudiante en los centros de prácticas}

Si nos apoyamos en la estructura de la asignatura de "prácticum" incluida en los actuales Planes de Estudios de los Grados universitarios, este tipo de tutoría debe constar de objetivos distribuidos en cuatro áreas de competencia: profesional, pedagógica y práctica, intercultural y lingüística. Cada una de estas áreas ha de constar, a su vez, de tres estadios consecutivos: observación, implementación (puesta en marcha) y evaluación (Varios, 2012). Las prácticas, desde esta perspectiva, tienen como meta fundamental establecer vínculos estrechos entre el conocimiento teórico, la observación, la participación y la toma de decisiones que corresponden a un profesional. La experiencia está configurada, por tanto, como un proceso de acción al que acompaña y sigue otro de reflexión, y que se continúa por un periodo de extracción de generalizaciones y de preparación para experimentar la siguiente experiencia (Zabalza, 2011).

Con respecto a la competencia profesional, y en cuanto a la observación, el estudiante debe concretar con el mentor los objetivos que se ha marcado cada uno, reflexionar sobre los códigos de conducta explícitos e implícitos del centro, examinar el papel que desempeñan los profesionales que participan en el mismo y entablar relaciones positivas con el mentor de acogida y otros profesionales del centro. En lo relativo a la implementación, el estudiante debe analizar su desarrollo profesional con el mentor de acogida y el tutor de la facultad, seguir sus consejos y tener en cuenta sus comentarios, así como establecer vínculos con los responsables y con los coordinadores del centro de cara a futuros proyectos profesionales. Y, en relación con la evaluación, debe comparar las reflexiones propias sobre la optimización de su competencia profesional presente y futura con las de la institución de acogida y con el tutor de la facultad, así como valorar las prácticas con su mentor, principalmente en lo que respecta a sus objetivos individuales, al desarrollo de su ética profesional y a las prácticas realizadas.

Con respecto a la competencia pedagógica y práctica y en cuanto a la observación, el estudiante debe reflexionar sobre cómo gestionar la organización de su trabajo, la relación entre sus miembros, la toma de decisiones y la distribución de responsabilidades, así como examinar los distintos métodos que se utilizan para supervisar, valorar y retroalimentar los resultados obtenidos. En lo relativo a la implementación, ha de preparar y participar en acciones que exijan una toma de decisión posterior con objetivos pedagógicos adecuados, demostrar en su práctica el conocimiento progresivo del entorno profesional en el que actúa y de sus competencias mediante el uso de recursos y métodos apropiados, al mismo tiempo que utilizar las evaluaciones de su práctica docente como base para mejorar planificaciones futuras. Y en lo que respecta a la evaluación, debe valorar de qué manera ha ido modificando los métodos de planificación, realización y control de sus acciones y comparar su enfoque de intervención con el utilizado en el centro de acogida. 
LA TUTORÍA UNIVERSITARIA EN EL ESCENARIO DEL ESPACIO EUROPEO DE EDUCACIÓN SUPERIOR: PERFILES ACTUALES

Con respecto a la competencia intercultural y en cuanto a la observación, el estudiante debe averiguar las características sociales y culturales del centro en el que se desarrollen sus prácticas, así como su enfoque de la diversidad. En lo relativo a la implementación, ha de dialogar con el tutor y los responsables del centro acerca de las medidas concretas que puedan fomentar el conocimiento cultural de todos los integrantes del mismo, aportar ideas para seleccionar y preparar materiales y recursos específicos que permitan incluir el componente multicultural en la actividad cotidiana del centro, supervisar la implantación de esos materiales y recursos y evaluar el impacto que han tenido en la vida del centro, así como comentar con el mentor de acogida cómo están modificando estas prácticas su percepción del papel de la diversidad cultural en la vida cotidiana del centro. Y en lo relativo a la evaluación, ha de analizar e identificar qué aspectos de su trabajo en la institución se han visto más afectados por la diversidad cultural del centro, además de explicar y justificar si ha modificado su enfoque, cuándo y por qué.

Con respecto a la competencia lingüística y en cuanto a la observación, el estudiante debe identificar el lenguaje específico para el funcionamiento de la organización, reconocer las propias necesidades y establecer prioridades referentes al lenguaje y la comunicación en la práctica, buscar y seleccionar recursos para mejorar la competencia lingüística y comunicativa y observar cómo utiliza la organización el idioma/lengua, tanto en situaciones formales como informales. En lo relativo a la implementación, ha de demostrar conocimientos avanzados del lenguaje que se utiliza en el grupo, la comunidad u organización correspondiente, ir dominando el lenguaje profesional a través de la interacción con el contexto, aplicar la propia competencia lingüística a diferentes escenarios para favorecer valores como la tolerancia, respeto, etc., e incorporar al propio registro lingüístico la diversidad sociolingüística de la organización. Y con relación a la evaluación, tiene que valorar con el mentor la diversidad lingüística y su uso en la actividad realizada en el centro y controlar en qué medida su desarrollo lingüístico le permite afrontar interacciones sociales y profesionales.

Desde esta perspectiva y según Marcelo y Estebaranz (1998), podemos referirnos a cuatro modelos de relación entre la universidad y los centros de prácticas. Cada uno de ellos le otorga un papel específicamente al mentor, es decir, al tutor que guía el aprendizaje del estudiante en prácticas en el centro de acogida.

a) Un modelo de yuxtaposición: cada cual en su sitio

Es el más tradicional, aquel que se basa en la observación de un modelo que después se imita, y en el que el estudiante establece las conexiones correspondientes entre la teoría y la práctica. En este modelo el profesional que es imitado es el mentor, siendo su papel fundamental el de un superior con más experiencia que enseña la práctica de una profesión con su ejemplo y forma de actuar. La mentoría del tutor es aquí espontánea, natural y no está planificada. 
LA TUTORÍA UNIVERSITARIA EN EL ESCENARIO DEL ESPACIO EUROPEO DE EDUCACIÓN SUPERIOR: PERFILES ACTUALES

b) Un modelo de consonancia en torno a un perfil de buen profesional

En este modelo se pretende que los profesionales con experiencia aprendan a trabajar como mentores, para ello interesa que tengan habilidad para cooperar como miembros de un equipo, que se comprometan con la introducción del estudiante en prácticas dentro de su profesión y que posean destrezas para reflexionar y articular sus ideas sobre la profesión. La universidad se plantea el objetivo de formar a los tutores de los centros de prácticas para que se conviertan en mentores, preparándoles para supervisar, guiar y apoyar las primeras experiencias reales de los estudiantes universitarios, promoviendo oportunidades de reflexión, estimulación y satisfacción en los mismos. La mentoría ejercida por el tutor, en este caso, tendría un carácter más formal.

\section{c) Un modelo de disonancia crítica: ver con otros ojos}

Este modelo se apoya en el aprendizaje experiencial y activo del estudiante en el centro de prácticas. Se otorga aquí al concepto de aprendizaje práctico una categoría epistemológica legitimada en la medida en que responde a las demandas de justificación y garantía de la misma forma que se exige al conocimiento formal. Y se realiza una diferenciación entre prácticas profesionales y práctica profesional. Las primeras representan ocasiones de inmersión a diferentes niveles de profundidad en situaciones o contextos característicos de la profesión a desempeñar. Las prácticas poseen sentido de realidad. En ellas, los estudiantes se aproximan a un mundo diferente que se plantea los problemas de la profesión de forma integrada. Las prácticas acercan al estudiante a la práctica, contribuyen a su aprendizaje profesional, pero no dejan de ser una simulación de la actividad profesional. Las prácticas se convierten, por lo tanto, en una ocasión para que los estudiantes realicen una autorreflexión, a través del autoanálisis, la supervisión de los compañeros o evaluación horizontal, así como mediante la orientación del mentor del centro de prácticas o evaluación vertical. A través de este modelo, los futuros profesionales se forman como investigadores, buscando respuestas a sus preguntas. Orientar la formación en este sentido exige tiempo para tareas de investigación, situaciones de búsqueda, diálogo y clarificación de preguntas y datos. Tareas éstas a cargo del mentor, que va construyendo su rol para ayudar al estudiante a desarrollar un estilo de práctica profesional reflexiva, a través de su autoanálisis como mentor. La mentoría aquí ejercida sería fundamentalmente situacional y de supervisión.

d) Un modelo de resonancia colaborativa: la formación como problema y responsabilidad compartida

Según este planteamiento, el proceso de formación práctica se entiende como un río al que van confluyendo experiencias, conocimientos, situaciones, que se producen en diferentes, pero igualmente válidos, contextos, tanto formales como informales, que conducen a la generación de un profesional cuya principal característica es que aprende a lo largo de la vida. Se entiende aquí que tanto la 
LA TUTORÍA UNIVERSITARIA EN EL ESCENARIO DEL ESPACIO EUROPEO DE EDUCACIÓN SUPERIOR: PERFILES ACTUALES

universidad como los centros de prácticas son espacios capaces de coordinarse para aprender y para permitir que los sujetos implicados aprendan. Se plantea, en definitiva, un modelo que debe generar un ambiente y una cultura de colaboración entre los miembros de ambas instituciones, a través de la realización de proyectos conjuntos y de la creación de redes de formación que ayuden a los sujetos implicados a aprender a cooperar (Marcelo y Estebaranz, 1998). Es un modelo en el que la mentoría se lleva a cabo de manera coordinada entre el tutor de la universidad y el tutor del centro de prácticas, siendo su responsabilidad compartida y su actividad de tutelaje complementaria. Se integran en este caso, tanto la mentoría natural, como la formal, la situacional y la de supervisión.

Estos modelos nos advierten de la necesidad de transformar la mentoría profesional en los centros de prácticas, pasando de una mera relación puntual y asilada, en un espacio y tiempo prefijados, hacia una relación constante y coordinada, basada en la responsabilidad compartida, en la relación auténtica entre teoría y práctica, así como en el aprendizaje permanente de todos los agentes implicados en este proceso.

- La tutoría del docente universitario experimentado hacia el docente novel

Una revisión de la literatura sobre el tema del aprendizaje docente nos regala algunas lecciones que es conveniente recordar:

1) Los conocimientos que adquirimos en la formación inicial tienen fecha de caducidad (Marcelo, 2002).

2) El conocimiento sobre la enseñanza no se construye individualmente, sino dentro de un espacio intersubjetivo (Pastré, 1994).

3) Es por naturaleza conocimiento basado en el contexto, conocimiento situado (McLellan 1996; Eraut, 1998).

4) Es conocimiento distribuido: por complejo, ningún profesor lo posee completo (Putnam y Borko, 2000).

Al mismo tiempo, hay que tomar conciencia de que los principios que inspiran el aprendizaje y la inserción profesional de los docentes no son neutros. Están ligados a los fines asignados a la universidad, que pueden ser: autonomía o conformismo; espíritu indagador o dogmatismo; reproducción o cambio social (Vélaz de Medrano, 2009).

La mentoría, en este sentido, consistiría en un proceso de acompañamiento en la tarea y la integración en el grupo e institución de referencia, y no en la mera actuación del mentor con respecto al mentorizado. Es una relación entre ambos, de reflexión compartida sobre problemas en los que los dos se ven inmersos e interpelados, un proceso de diálogo profesional en el que la mayor experiencia es uno de los principales rasgos que configuran el rol del mentor (Vélaz de Medrano, 2009). 
LA TUTORÍA UNIVERSITARIA EN EL ESCENARIO DEL ESPACIO EUROPEO DE EDUCACIÓN SUPERIOR: PERFILES ACTUALES

Y es además una actividad particularmente orientada al desarrollo profesional, por lo que reviste interés recordar cuáles son, desde una perspectiva macro, las dimensiones y factores que la configuran en mayor medida (Vélaz de Medrano, 2008):

- El espacio profesional (la "Ocupación").

- La formación inicial y permanente (las "competencias").

- El modo de acceso a la profesión (especialmente a las profesiones reguladas).

- Las funciones y tareas (prescritas en las normas, demandadas, deseadas por el profesional, etc.).

- La promoción.

- El pensamiento del sujeto sobre su tarea profesional (cómo se la "representa").

- Las condiciones laborales (salario, recursos).

- La organización profesional (pertenencia a asociaciones y colegios profesionales).

- La generación y difusión del conocimiento profesional (investigación, innovación y publicación).

- El espacio social, es decir, la consideración social de una profesión y la forma en que los demás actores del sistema representan el rol de unos u otros profesionales.

Si asumimos que todos ellos son factores significativos del desarrollo profesional, el contexto de trabajo (en este caso universitario) es uno de los que en mayor medida acaba delimitando el espacio profesional propio y, también y no menos importante, el espacio de colaboración multiprofesional. Es por ello que la mentoría debe estar estrechamente ligada al contexto.

Y si asumimos, desde una perspectiva micro, que el desarrollo profesional docente es la capacidad del profesorado para mantener la curiosidad acerca de la clase, identificar intereses significativos en el proceso de enseñanza-aprendizaje, así como valorar y buscar el diálogo con colegas expertos como apoyo a su labor docente (Marcelo, 2002), uno de los principales objetivos de la mentoría será fomentar en el principiante una actitud permanente de indagación, de afrontar problemas como retos profesionales y no como riesgos personales, y de formular soluciones tentativas, debatirlas y contrastarlas. La mentoría tiene como fin ayudar a construir o mejorar el conjunto de competencias intelectuales, personales, sociales y técnicas que el docente novel ha de poner en juego para que sus estudiantes aprendan (Vélaz, 2009).

Tras estas reflexiones cabría plantearse a continuación la existencia de diferentes tipos de mentoría, en función del número de mentores, del número de mentorizados y del perfil del mentor.

- Por el número de mentores, nos referimos a si la responsabilidad de desarrollar un proceso de mentoría recae sólo en un docente experimentado 
LA TUTORÍA UNIVERSITARIA EN EL ESCENARIO DEL ESPACIO EUROPEO DE EDUCACIÓN SUPERIOR: PERFILES ACTUALES

sobre otro novel (por ejemplo, un catedrático respecto de un ayudante) o en un conjunto de docentes sobre un mentorizado (por ejemplo, los docentes de un departamento universitario en el que está inscrito el ayudante). Si la mentoría tiene carácter individual, la ventaja es que la relación es más personal y continuada, y el seguimiento es mayor. Pero existe el inconveniente de que si la figura del mentor no es la más adecuada, el mentorizado en realidad no se integra completamente en la institución educativa. Si, en cambio, el mentorizado es tutorado por un conjunto de profesores es posible que adquiera un aprendizaje más amplio y diversificado, aunque si ese profesorado es muy dispar o no tiene un proyecto de enseñanza común, el principiante puede llegar a sufrir una «esquizofrenia docente".

- Por el número de mentorizados, nos referimos a si el proceso de mentoría se realiza desde un docente experimentado hacia uno novel o desde un mentor a un conjunto de mentorizados. En el primer caso, abundamos en el razonamiento anterior y en el segundo, la ventaja de un proceso de uno a varios es que se aprovecha más el aprendizaje y es posible, además, que se produzcan diferentes visiones de la práctica docente. Sin embargo, puede llegar a provocar enfrentamiento entre los mentorizados o un mal aprendizaje que afecte a varios principiantes al mismo tiempo.

- $\quad$ Por el perfil del mentor, nos referimos a la tutoría que ejerce un docente experimentado sobre uno novel, en materia pedagógica, es decir, en cuanto a cómo debe desarrollarse el proceso de enseñanza-aprendizaje en el aula. O al conocimiento y habilidad técnica poseída por el mentor y orientada a favorecer en el principiante un desarrollo específico y experto en la materia a impartir. Ambos deben darse y ambos son valiosos, aunque tal vez más el primero, pues el segundo puede ir adquiriéndose por el propio mentorizado, necesitando más un ejemplo didáctico.

En un proceso de mentoría de estas características, además de todo lo señalado hasta el momento, es necesario que el mentor posea unas competencias específicas para poder realizar un acompañamiento al principiante de manera efectiva (Vélaz de Medrano, 2009):

- Competencia cognitiva: empleo de teorías y conceptos obtenidos a través del estudio, así como del conocimiento tácito e informal adquirido por la experiencia; movilizando esos conocimientos, integrándolos para comprender la realidad y transfiriéndolos a diferentes situaciones (Coll y Martín, 2006).

- Competencia investigadora: profundización, ampliación, recreación y depuración del conocimiento, haciendo partícipe al mentorizado, iniciándole en este campo y suscitándole interés por él (García Nieto, 2008). 
LA TUTORÍA UNIVERSITARIA EN EL ESCENARIO DEL ESPACIO EUROPEO DE EDUCACIÓN SUPERIOR: PERFILES ACTUALES

- Competencia tecnológica: que se traduce en habilidades instrumentales de dominio y uso de nuevos recursos y lenguajes (audiovisual, informático, digital...), que deben transferirse al mentorizado.

- Competencia pedagógica (habilidades y saber hacer): conocimiento de las particularidades del aprendizaje adulto y de las metodologías de enseñanza más adecuadas, generando relaciones de confianza, promoviendo el aprendizaje activo, proporcionando feedback y actuando como modelo.

- Competencia interpersonal: se pone el énfasis en la relación, emplea un enfoque facilitador, la empatía y la asertividad.

- Competencia ética: implica un comportamiento coherente con un conjunto de valores personales y profesionales.

- Competencia metacognitiva (toma de conciencia y autorregulación): posibilita un aprendizaje autónomo para seguir aprendiendo a lo largo de la vida (Coll y Martín, 2006).

De acuerdo con este planteamiento, un profesional competente -en nuestro caso, el mentor- sería aquel que conoce y regula sus propios procesos de construcción del conocimiento, tanto desde el punto de vista cognitivo como emocional, y puede hacer un uso estratégico de los mismos, ajustándolos a las circunstancias específicas del problema o situación a la que se enfrenta.

Si apoyamos estos planteamientos en experiencias prácticas realizadas en este sentido, descubrimos la existencia de tres posibles vías para concretar un enfoque de estas características (Vieluf et al., 2012):

1. Los programas de inserción: algunos programas de iniciación incluyen, entre sus actividades, el asesoramiento de los docentes principiantes por medio de otros profesores, que pueden ser compañeros o bien "mentores" (Vaillant y Marcelo, 2001). En estos programas, la figura del mentor debe ser la de un docente con dedicación permanente, con experiencia didáctica, con habilidad en la gestión del aula, disciplina, comunicación con los colegas, con conocimiento del contenido de la asignatura que imparte, con iniciativa para planificar y organizar y con cualidades personales (flexibilidad, paciencia, sensibilidad). Un curioso ejemplo que nos parece oportuno incluir en este apartado es el propuesto por Gordon y Brobeck (2010), quienes desarrollan un programa de mentoring destinado a profesores experimentados para que ejerzan, con posteridad, una labor de mentores con profesores noveles.

2. El aprendizaje relacionado con la práctica: Cochran-Smith y Lytle (1999) clasifican la investigación sobre aprender a enseñar, tomando como criterio las relaciones que se establecen entre el conocimiento que se produce y su aplicación en la práctica de la enseñanza. De este modo, diferencian entre el conocimiento para la práctica, que tiene carácter formal, se deriva de la investigación universitaria y se apoya en una metodología deductiva (de la teoría podemos derivar la aplicación práctica); el conocimiento 
LA TUTORÍA UNIVERSITARIA EN EL ESCENARIO DEL ESPACIO EUROPEO DE EDUCACIÓN SUPERIOR: PERFILES ACTUALES

en la práctica o conocimiento en la acción, que se adquiere mediante la experiencia y la reflexión sobre la misma (Feiman-Nemser, 2001); y el conocimiento de la práctica, más inductivo, que permite al docente contextualizar sus aprendizajes. En este sentido, el uso de los One Minute Papers supone un medio de bajo coste, sencillo y de demostrada eficiencia para aumentar la interrelación entre profesorado y alumnado, mejorar la satisfacción de los estudiantes y de los docentes, así como optimizar los resultados académicos ${ }^{3}$. Este ejemplo nos aporta una experiencia de aprendizaje basado en la práctica (de carácter deductivo, aplicativo y con proyección inductiva), en el que se combina una mentoría procedente del profesorado experimentado, como promotor de la idea, con la mentoría de los propios estudiantes, quienes a través de su intervención en el aula facilitan al docente una guía para la mejora y reconducción de su práctica profesional (León y García, 2010).

3. El aprendizaje a través de redes: el término red se entiende como una malla de personas conectadas por enlaces en torno a los cuales fluyen cosas como objetos, trabajo, afectos, evaluación, conocimiento, prescripciones, influencia y poder, y en las que la mayoría de los participantes están conectados unos con otros. Las redes enlazan a diferentes personas con distintos propósitos, utilizando una variedad de fórmulas (Marcelo, 2001). Lieberman y Grolnick (1996) entienden que las redes constituyen una forma de implicar al profesorado en la dirección de su propio aprendizaje; le permiten superar las limitaciones de su rol institucional, jerarquía y localización geográfica, y le animan a trabajar con personas diferentes. Los participantes tienen, así, la oportunidad de crecer y desarrollarse en una comunidad profesional que se centra en su propio perfeccionamiento, proporcionando formas de aprendizaje más relacionadas con las experiencias profesionales vividas. Por lo tanto, en palabras de Day (1999), las redes proporcionan estructuras organizativas que permiten a los grupos de docentes reunirse para hablar de su trabajo, aprender de otros y resolver temas curriculares y estructurales. Las redes configuran espacios en los que el aprendizaje está distribuido, donde es posible construir colaborativamente y donde el profesorado es el protagonista y responsable de su formación. Un ejemplo de carácter europeo que clarifica las

3. Los orígenes del One Minute Paper no están muy claros. Las primeras bibliografías que incluyen el término proceden de los años ochenta y noventa. Sin embargo, el uso generalizado entre el profesorado y su difusión se originó en la Universidad de Harvard, Massachusetts, como resultado de un proyecto de mejora de la calidad docente propuesto en 1986 por el profesor Derek Bok, decano de la Facultad de Derecho y primer presidente de la Universidad de Harvard, denominado "The Harvard Assessment Seminar" (Light, 1990). Esta técnica se ha introducido con posterioridad en numerosas universidades internacionales como Berkeley, Virginia, Denver, Otawa, etc.; así como en universidades españolas como la de Zaragoza, Valencia o Santiago de Compostela (LEón y GARCíA, 2010). 
LA TUTORÍA UNIVERSITARIA EN EL ESCENARIO DEL ESPACIO EUROPEO DE EDUCACIÓN SUPERIOR: PERFILES ACTUALES

ideas precedentes es la red ETwinning (Css, 2011). Proyecto que se lanzó en el año 2005 y se renovó en el 2008, y que se concibe como una red social por Internet, en la que los docentes se inscriben, se conectan a su escritorio e inmediatamente comienzan a interconectarse, leen los éxitos conseguidos por otros, mandan y cuelgan mensajes en el foro y hacen nuevos contactos con colegas de toda Europa.

\section{A MODO DE CONCLUSIÓN}

En el presente trabajo nos apoyamos en la concepción de la tutoría universitaria como la actividad sistémica, intencional, correctamente articulada y programada, que hay que dotar de una estructura de funcionamiento y hay que concederle un lugar destacado en la planificación de las actividades académicas propias del quehacer de la universidad. Se caracteriza fundamentalmente por facilitar e impulsar el desarrollo integral de los universitarios, personalizar la educación y encaminar al universitario hacia la madurez personal y el crecimiento intelectual y científico. Integra la dimensión intelectual-cognitiva, afectivo-emocional y socioprofesional. Y se sustenta en modelos distintos según su objetivo: teaching (tutoría académica), counseling (tutoría personal) y career advising (tutoría profesional).

El perfil del tutor que se deduce del concepto precedente y que se enmarca en el actual Espacio Europeo de Educación Superior lleva aparejadas cualidades humanas (empatía, autenticidad, madurez, responsabilidad y sociabilidad) y cualidades científico-técnicas (capacidad de planificación, organización, coordinación, motivación, evaluación, así como dominio técnico y psicopedagógico).

El tutor, dentro de este contexto, puede jugar diferentes papeles en el entorno universitario:

- El docente como coach, que facilita una experiencia formativa sustentada en la confianza entre el tutor y el estudiante y busca la dinamización y el asesoramiento de un docente hacia un estudiante.

- La tutoría entre iguales o mentoría de pares, la cual, partiendo de la consideración del mentoring como la orientación al desarrollo de las capacidades, competencias y actitudes del otro, más acordes con su potencial, pretende favorecer la adaptación a la institución universitaria del alumnado de nuevo acceso. Se presenta como una buena estrategia para promover conjuntamente competencias generales y específicas en un contexto de aprendizaje colaborativo y dentro de un clima de confianza.

- La tutoría o mentoring profesional del estudiante en los centros de prácticas, que se desarrolla a través de dos tipos de profesionales: el tutor de la universidad, quien ejerce un papel de apoyo y seguimiento, y el mentor del centro de acogida, que puede ejercer una mentoría espontánea y no planificada (modelo de yuxtaposición), una mentoría formal (modelo de consonancia profesional), una mentoría situacional y de supervisión 
LA TUTORÍA UNIVERSITARIA EN EL ESCENARIO DEL ESPACIO EUROPEO DE EDUCACIÓN SUPERIOR: PERFILES ACTUALES

(modelo de disonancia crítica) o una mentoría combinada (modelo de resonancia colaborativa).

- La tutoría o mentoría de un docente universitario experimentado hacia un docente novel, la cual consistiría en un proceso de acompañamiento en la tarea y la integración en el grupo e institución de referencia. Planteamos tres vías diferentes pero complementarias para su desarrollo: los programas de inserción, el aprendizaje relacionado con la práctica y el aprendizaje a través de redes.

En definitiva, en este artículo hemos pretendido identificar el concepto de tutoría universitaria que reclama el EEEs, definiendo el perfil de quien ejerce esa tutoría e identificando los diferentes papeles que dicho profesional puede y debe jugar según el contexto de referencia, el individuo receptor y, evidentemente, los objetivos propuestos.

\section{REFERENCIAS BIBLIOGRÁFICAS}

ALBA, E. (2009) La formación de tutores en coaching y mentoring en la Universidad Europea de Madrid, en JIMCUE 209-IV Jornadas Internacionales Mentoring \& Coaching: Universidad-Empresa, 23, 24 y 25 de noviembre. Madrid, 235-249.

AREA, M.; SAN Nicolás, M. a B. y FariÑa, E. (2010) Buenas prácticas de aulas virtuales en la docencia universitaria semipresencial. TESI (Teoría de la Educación en la Sociedad de la Información), 11 (3), 7-31.

Argos, J.; García del Dujo, Á.; Romero, C. y Vera, J. (2011) Autonomía y responsabilidad en el contexto de la escuela. Ponencia presentada al XXX Seminario Interuniversitario de Teoría de la Educación, bajo el título "Autonomía y responsabilidad. Contextos de aprendizaje y educación en el siglo XXI". Barcelona. Octubre.

CANO, R. (2008) La tutoría universitaria en el Espacio Europeo de Educación Superior. Revista Interuniversitaria de Formación del Profesorado, 22 (1), 17-20.

CANO, R. (2009) Tutoría universitaria y aprendizaje por competencias. ¿Cómo lograrlo? Revista Electrónica Interuniversitaria del Profesorado, 12 (1), 181-204.

Cochran-SMith, M. y LYTLE, S. (1999) Relationships of knowledge and practice: Teacher learning in communities. Review of Research in Education, 24, 249-305.

http://dx.doi.org/10.2307/1167272.

http://dx.doi.org/10.3102/0091732X024001249.

Coll, C. y MARTín, E. (2006) The prevalence of the curriculum debate. Basic learning, competencies, and standards. PRELAC Journal, 3, 6-27.

CSs (2011) Etwinning 2.0. Hacia la comunidad de centros escolares en Europa. Bruselas, European Schoolnet.

DAY, C. (1999) Developing Teachers. The challenges of Lifelong Learning. London, Falmer Press.

Delors, J. (1997) La educación encierra un tesoro. París, UnESCO.

Díaz, F. (2000) Mentoring: Una relación de confianza. Capital Humano, 137, 72-74.

ERAUT, M. (1998) Concepts of Competence. Journal or Interprofessional Care, 12 (2), 127-139. http://dx.doi.org/10.3109/13561829809014100. 
LA TUTORÍA UNIVERSITARIA EN EL ESCENARIO DEL ESPACIO EUROPEO DE EDUCACIÓN SUPERIOR: PERFILES ACTUALES

Feiman-Nemser, S. (2001) From preparation to practice: Designing a continuum to strengthen and sustain teaching. Teachers College Record, 103 (6), 1013-1055.

http://dx.doi.org/10.1111/0161-4681.00141.

FERnÁNDEZ-SAlinERO, C. (2008) Modalidades de formación en las organizaciones, en PINEDA, P. (coord.) La gestión de la formación en las organizaciones. Barcelona, Ariel, 115-148.

García Nieto, N. (2008) La función tutorial de la Universidad en el actual contexto de la Educación Superior. Revista Interuniversitaria de Formación del Profesorado, 22 (1), 21-48.

Gordon, S. P. y Brobeck, S. R. (2010) Coaching the Mentor: Facilitating Reflection and Change. Mentoring \& Tutoring: Partnership in Learning, 18 (4), 427-447. http://dx.doi.org/10.1080/13611267.2010.511851.

Hashuel, P. (2010) El coaching llega a la educación. Bilbao, Desclée de Brouwer.

Hernández Franco, V. y TORRES, J. (2005) La acción tutorial en la Universidad. Informe técnico. Madrid, Universidad Pontificia de Comillas.

LEÓN, A. y GarCíA, L. (2010) El "One Minute Paper" como elemento eficaz y asequible para la mejora de la calidad docente en el Espacio Europeo de Educación Superior, en LEÓN, M. J. y LÓPEZ, M. C. (coords.) Formación Inicial del profesorado universitario. El papel de los mentores. Granada, Vicerrectorado para la Garantía de la Calidad. Secretariado de Formación y Apoyo a la Calidad, 134-142.

Lieberman, A. y Grolnick, M. (1996) Networks and Reform in American Education. Teacher College Record, 98 (1), 7-45.

Light, R. (1990) The Harvard Assessment Seminars. Cambridge, Mass., Harvard University.

MARCElo, C. (ed.) (2001) La función docente. Madrid, Síntesis.

Marcelo, C. (2002) Learning to Teach for a Knowledge Society. Education Policy Analysis Archives, 10 (35), 1-52.

Marcelo, C. y Estebaranz, A. (1998) Modelos de colaboración entre la Universidad y las escuelas en la formación inicial del profesorado. Revista de Educación, 317, 97-120.

Mariano, R. de (1999) Un cuento de management y coaching. Capital Humano, 127, $28-32$.

MarTínez GonzÁlez, J. A. (2011) La orientación y la tutoría en el Espacio Europeo de Educación Superior. Cuadernos de Educación y Desarrollo, 23 (3), 1-39.

MCLellan, H. (1996) Creating Virtual Communities Via the Web, en KaHN, B. (ed.) Web-based Instruction. New Jersey, Englewood Cliffs, 185-190.

Michavila, F. y García Delgado, J. (eds.) (2003) La tutoría y los nuevos modos de aprendizaje en la Universidad. Madrid, Comunidad de Madrid, Dirección General de Universidades.

Mosca, A. y SANTiago, C. (2012) Fundamentos conceptuales de las tutorias entre pares. La experiencia de la Universidad de la República. Montevideo, Universidad de la República.

Novo, M. (2007) El desarrollo sostenible. Su dimensión ambiental y educativa. Madrid, uNESCO-Prentice Hall.

Pastré, P. (1994) Variations sur le développement des adultes et leurs representations. Éducation Permanente, 119, 33-63.

Putnam, R. T. y Borko, H. (2000) El aprendizaje del profesor: Implicaciones de las nuevas perspectivas de la cognición, en BidDle, B. J.; GoOD, T. L. y Goodson, I. F. (eds.) La enseñanza y los profesores (I). La profesión de enseñar. Barcelona, Paidós, 219-309.

Risquez, A. (2011) Peer Electronic mentoring for transition into university: A theoretical review. Revista Española de Orientación y Psicopedagogía-REOP, 22 (3), 232-239. 
LA TUTORÍA UNIVERSITARIA EN EL ESCENARIO DEL ESPACIO EUROPEO DE EDUCACIÓN SUPERIOR: PERFILES ACTUALES

Rodríguez Espinar, S. (coord.) (2004) Manual de tutoría universitaria. Recursos para la acción. Barcelona, Octaedro.

Rogers, C. R. (1975) El proceso de convertirse en persona. Barcelona, Paidós.

Rowley, J. B. (1999) The good mentor. Educational Leadership, 56 (8), 20-22.

Ruiz Corbella, M.; Escámez, J.; Bernal, A. y Gil, F. (2011) Autonomía y responsabilidad en los contextos socioeducativos del siglo XXI. Ponencia presentada al XXX Seminario Interuniversitario de Teoría de la Educación, bajo el título "Autonomía y responsabilidad. Contextos de aprendizaje y educación en el siglo XXIm. Barcelona. Octubre.

Sánchez Ávila, C.; Macías, J.; Almendra, A. y Jiménez Leube, F. J. (2007) Origen, desarrollo, evolución y resultados del Proyecto Mentor en la ETSI de Telecomunicaciones de la Universidad Politécnica de Madrid. Cuaderno de Innovación Educativa en las Enseñanzas Técnicas Universitarias, 1 (2), 35-46.

Vaillant, D. y Marcelo, C. (2001) Las tareas del formador. Málaga, Aljibe.

Valverde, A.; Ruiz de Miguel, C.; García Jiménez, E. y Romero, S. (2003-2004) Innovación en la orientación universitaria: La mentoría como respuesta. Contextos Educativos, 6-7, $87-112$.

VV. AA. (2012) Guía de prácticas del Grado en Pedagogía. Madrid, Facultad de Educación (UCM).

Velasco, P. J. y Benito, Á. (2011) Peer mentoring at the Universidad Europea de Madrid: An educational strategy for the development of general and specific competences. Higher Learning Research Communications, 1 (1), 22-32.

Vélaz DE Medrano, C. (2008) Formación y profesionalización de los orientadores desde el enfoque de competencias. Educación XXI, 11, 155-181.

Vélaz de Medrano, C. (2009) Competencias del profesor-mentor para el acompañamiento al profesor principiante. Profesorado. Revista de Curriculum y Formación del Profesorado, 13 (1), 210-229.

Vieluf, S.; Kaplan, D.; Klieme, E. y Bayer, S. (2012) Teaching Practice and Pedagogical Innovations. Evidence from TALIS (Teaching and Learning International Survey). Paris, OECD Publishing.

Zabalza, M. Á. (2011) El Prácticum en la formación universitaria: estado de la cuestión. Revista de Educación, 354, 21-43. 